\title{
19. COMMISSION DE LA ROTATION DE LA TERRE
}

Président: B. Guinot.

SECRÉTAIRE: R. Vicente.

\author{
Comptes-rendus des séances
}

La commission a tenu 4 séances, les 23,25, 26 et 28 août 1967.

I. BIH

Il est annoncé que tous les problèmes touchant le BIH seront discutés lors des réunions de la commission 31 .

\section{SYMPOSIUM UAI 32 ET RÉSOLUTIONS DU COMITÉ NATIONAL DE GÉODÉSIE D'URSS}

W. Markowitz présente les résolutions adoptées par le symposium 32 tenu à Stresa en mars 1967 dont le sujet était: mouvement séculaire du pôle, dérive des continents, rotation de la Terre. Le texte de ces résolutions apparaît dans les comptes-rendus de ce symposium. Une des résolutions proposait le choix du 'nouveau pôle de 1900-05' de Cecchini comme pôle-origine, une autre la constitution de nouvelles chaînes d'instruments destinées à éliminer les erreurs introduites par les catalogues d'étoiles.

A.A. Mihailov présente ensuite un rapport du Comité National de Géodésie d'URSS relatif également à la constitution de chaînes d'instruments sur des parallèles communs: chaîne de PZT sur le parallèle international $+39^{\circ}$, chaînes de PZT et d'astrolabes à installer dans l'hémisphère austral.

Comme le montre le rapport du groupe de travail constitué à Stresa, la synthèse des points de vue sur les chaînes d'instruments fut aisément réalisée (recommandation 1).

A propos de l'origine du pôle, E.P. Fedorov s'oppose à la résolution de Stresa car, selon lui, on ne peut pas affirmer qu'il existe réellement une dérive du pôle géographique et parce que, pour certains travaux de géophysique, le pôle 'fixe' est, en fait, le pôle défini par le petit axe d'inertie de la Terre. $P$. Melchior préférerait l'appellation 'pôle origine' au lieu de pôle fixe. E.P. Fedorov, cependant, reconnait que pour des raisons de commodité, conformes aux traditions astronomiques, on peut exprimer les coordonnées du pôle instantané dans un système défini par les latitudes conventionnelles des 5 stations du parallèle $+39^{\circ}$, sans mentionner que ce système définit un pôle à une date quelconque. Ce point de vue est adopté unanimement par la commission (résolution 1).

\section{RÉSOLUTION PROPOSÉE PAR LE COMITÉ NATIONAL BELGE SUR LES NUTATIONS À COURTE PÉRIODE ET LES MARÉES TERRESTRES}

Un exposé de synthèse présenté par $P$. Melchior montre comment les perturbations extérieures exercées sur la Terre donnent lieu simultanément à la précession et aux nutations de l'axe de rotation dans l'espace, aux nutations de l'axe de rotation par rapport à la Terre et aux marées terrestres et insiste sur l'intérêt de ne pas dissocier l'étude de ces divers phénomènes.

La commission 19, pleinement consciente de la valeur des travaux de P. Melchior et de ses collaborateurs a unanimement recommandé que ces travaux soient poursuivis et étendus; elle a approuvé dans son principe une résolution soumise par le Comité National Belge à l'Assemblée Générale qui proposait l'examen de ces phénomènes, mais elle en a légèrement modifié la forme à la demande de P. Melchior et W. Markowitz (résolution A2).

\section{RAPPORTS DU SILET DU SIMP}

T. Nicolini a définitivement calculé les coordonnées du pôle pour la période 1941-1949. Le volume 
$\mathrm{X}$ des résultats du SIL est sous presse. Les coordonnées du pôle publiées dans le vol. XIIIA des Transactions de l'UAI ont pu être améliorées par l'usage de séries d'observations discontinues de Carloforte et Kitab. Voir Annexe 1.

S. Yumi présente le rapport sur les travaux du SIMP. Les résultats préliminaires paraissent dans les rapports annuels auxquels on voudra bien se reporter. Les coordonnées du pôle de 1962.00 à 1966.35 ont été publiées dans le volume XIIIA; on donne (Annexe II) les coordonnées de 1966.00 à 1967.20. Les coordonnées de 1966.00 à 1966.35 ont été très légèrement modifiées.

La commission exprime unanimement ses remerciements à $T$. Nicolini et $S$. Yumi.

Nous n'avons pas reçu d'informations plus récentes que celles publiées dans le vol. XIIIA sur la publication des résultats de la période 1949-1962.

\section{PRÉSENTATION DE TRAVAUX SCIENTIFIQUES}

E.P. Fedorov rappelle les traits caractéristiques des séries de latitude:

(1) il est impossible de caractériser la précision des observations par un seul nombre, la précision étant fonction de la fréquence;

(2) les erreurs accidentelles peuvent simuler des variations périodiques.

Il présente ensuite les travaux de ses collaborateurs:

(a) I.I. Glagoleva et Ya.S. Yatskiv ont développé pratiquement une méthode d'analyse qui exclut tout arbitraire;

(b) S.P. Major a réduit à un système commun les mesures de latitude de Mizusawa, Carloforte et Ukiah, de 1900 à 1934 et les a analysées, il donne une liste des termes périodiques trouvés;

(c) V.K. Tarady a tenté de déduire les termes principaux de la nutation de l'analyse des observations du SIL, l'accord entre les valeurs observées et théoriques n'est pas satisfaisant.

S. Débarbat a analysé les mesures de temps et de latitude faites à Paris depuis 1956.5 jusqu'à 1963.0 avec l'astrolabe Danjon; elle en a déduit les variations lunaires de la verticale et a mis en évidence le terme de nutation diurne prévu par Molodensky et déjà trouvé par d'autres auteurs.

$S$. Yumi a présenté les travaux des astronomes de l'observatoire de Mizusawa: (a) T. Okuda, d'après l'étude des termes $Z$, est conduit à penser qu'il pourrait exister des variations globales du potentiel de la Terre. (b) Y. Wako suppose que le terme $\mathrm{Z}$ est dû à des termes erronés de la nutation; (c) M. Kirita montre que le mouvement annuel du pôle est dû principalement à la circulation atmosphérique.

$N$.S. Sidorenkov estime que la circulation atmosphérique rend compte des irrégularités à longue période; il attire l'attention sur la nécessité d'avoir des données météorologiques plus précises et plus complètes. -

M. Torao présente l'étude de S. Iijima et $\mathrm{S}$. Okazaki sur la composante biannuelle de la vitesse de rotation de la Terre. Cet effet, d'amplitude totale $18 \mathrm{~ms}$, est attribué aux vents stratosphériques.

$N$. Stoyko discute d'abord de la précision des coordonnées du pôle instantané pour la période 1941-1949. Il étudie ensuite les diverses méthodes de représentation de la rotation de la Terre: ces méthodes ne permettent pas une extrapolation. Cependant la corrélation entre la vitesse de la rotation et la longitude des noeuds de la lune, de Mme A. Stoyko semble se confirmer.

$A$. Stoyko a trouvé de fortes corrélations entre l'activité du Soleil, la nutation libre, la vitesse de rotation de la Terre, la circulation générale de l'atmosphère et l'énergie des séismes profonds.

$H$. Enslin étudie les méthodes pour calculer les corrections de groupes. Ces diverses méthodes donnent des résultats équivalents pour un programme donné. Mais il y a intérêt à choisir le programme qui correspond le mieux au but recherché, il y a en particulier, intérêt à réduire le nombre des groupes d'un programme pour éliminer les erreurs en fonction de l'ascension droite.

H.M. Smith présente le projet d'astrolabe à réflexion de D. Thomas. La réflexion sur un prisme de CER-VIT permettrait d'éliminer les effets thermiques sur la distance zénithale, constatés sur les astrolabes Danjon et de choisir une distance zénithale instrumentale quelconque. Les dimensions de l'instrument pourraient être accrues. Un montage expérimental sur un astrolabe Danjon est en cours. 
Annexe I. Coordonnées du pôle de 1941 à 1949 (valeurs révisées), rapportées à l'origine conventionnelle internationale unité 0". 001

Fraction d'année

$$
1941
$$

1942

1943

1944

$\begin{array}{lll} & x & y \\ 060 & +66 & +65 \\ 143 & +45 & +37 \\ 226 & +22 & +90 \\ 310 & -15 & +101 \\ 393 & +8 & +134 \\ 476 & +29 & +177 \\ 560 & +69 & +196 \\ 643 & +172 & +209 \\ 726 & +137 & +192 \\ 810 & +129+154 \\ 893 & +148+97 \\ 976 & +144+83\end{array}$

\begin{tabular}{|c|c|}
\hline & \\
\hline+156 & +62 \\
\hline & +74 \\
\hline+7 & +45 \\
\hline & +113 \\
\hline 3. & +100 \\
\hline+ & +110 \\
\hline+2 & +113 \\
\hline+ & +112 \\
\hline & +171 \\
\hline 1 & +170 \\
\hline 3 & +172 \\
\hline-2 & +225 \\
\hline
\end{tabular}

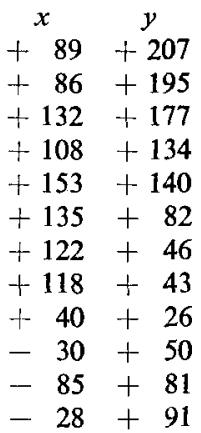

\begin{tabular}{|c|c|}
\hline & \\
\hline 11 & + \\
\hline & \\
\hline 2 & \\
\hline & \\
\hline 19 & \\
\hline 28 & \\
\hline 3 & \\
\hline $29 ?$ & \\
\hline 16 & \\
\hline 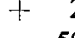 & \\
\hline & \\
\hline
\end{tabular}

Fraction d'année

060
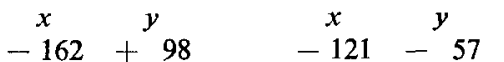

$x$
$+103-75$

$\begin{array}{cc}x & y \\ +264 & +108\end{array}$

$-158+131$

$-189+2$

$\begin{array}{r}+103-75 \\ +\quad 7-77 \\ \hline\end{array}$

$+237+7$

$-113+238$

$-184+86$

$-76-28$

$+191-19$

226
310

$-53+301$
$+94+310$

$-145+203$

$-150+91$

$+106-62$

393

$+200+311$

$-74+285$

$-154+160$

$+17-23$

476

$+62+330$

$-84+246$

$-32+27$

$+266+172$

$+174+346$

$+10+285$

$-29+67$

643

726

$+286+105$

$+219+324$

$+119+324$

$-38+139$

$+329+10$

$+254+268$

$+189+311$

$-78+184$

$+252-95$

$+309+122$

$+263+272$

$-32+253$

893

$+114-137$

$+323-30$

$+286+227$

$+26+305$

976

$+64-104$

$+233-40$

$+278+148$

$+129+324$

Annexe II. Coordonnées du pôle de 1966.00 à $1967 \cdot 20$ rapportées à l'origine conventionnelle internationale unité 0". 001

\begin{tabular}{|c|c|c|c|c|c|c|c|c|}
\hline $\begin{array}{c}\text { Date } \\
\text { de Bessel }\end{array}$ & $x$ & $y$ & $\begin{array}{c}\text { Date } \\
\text { de Bessel }\end{array}$ & $x$ & $y$ & $\begin{array}{c}\text { Date } \\
\text { de Bessel }\end{array}$ & $x$ & $y$ \\
\hline $1966 \cdot 00$ & +75 & +114 & $1966 \cdot 40$ & -119 & +215 & $1966 \cdot 85$ & +125 & +291 \\
\hline 05 & $\begin{array}{r}+33 \\
+\quad 33\end{array}$ & +103 & 45 & -115 & +255 & 90 & +123 & +273 \\
\hline 10 & 0 & +98 & 50 & -104 & +298 & 95 & +115 & +253 \\
\hline 15 & -29 & +100 & 55 & -86 & +330 & 1967.00 & +99 & +233 \\
\hline 20 & -58 & +108 & 60 & -57 & +344 & 05 & +79 & +213 \\
\hline 25 & -86 & +124 & 65 & -10 & +345 & 10 & +56 & +194 \\
\hline 30 & -105 & $\begin{array}{r}+149 \\
\end{array}$ & 70 & $\begin{array}{r}15 \\
+\quad 52\end{array}$ & +337 & 15 & $\begin{array}{l}+31 \\
\end{array}$ & +177 \\
\hline 35 & -116 & +181 & $\begin{array}{l}75 \\
80\end{array}$ & $\begin{array}{l}+96 \\
+117\end{array}$ & $\begin{array}{r}+324 \\
+\quad 308\end{array}$ & 20 & +12 & +165 \\
\hline
\end{tabular}




\section{Annexe III. Complément au rapport du vol. XIIIA}

\section{INFORMATION ON THE LATITUDE OBSERVATIONS OF THE PULKOVO OBSERVATORY}

Latitude observations were renewed at Pulkovo in 1948 when the old zenith-telescope of $135 \mathrm{~mm}$ aperture was put in operation after the rebuilding of the observatory during the war. The program of observation compiled for a whole period of nutation was completed in 1967. During this time 47.4 thousand determinations of latitude were made. Beginning from 1955 the results were communicated to the SIL and SIR. In 1955-61 the observations were made on an extended program lasting through the whole night (in winter up to 18 hours), in order to determine latitude variations of short period. These observations are reduced with the new value of the constant of aberration and taking into account Oppolzer's terms and the tidal deflection of the vertical. Much attention was paid to the determination of the value of the screw.

In order to determine the second coordinate of the pole's motion a new latitude station was organized at Blagoveshchensk in the Far East, $97^{\circ}$ east of Pulkovo. Regular observations with a zenith-telescope of $180 \mathrm{~mm}$ aperture were begun in 1959. From 1959.17 to 1967.42, 19528 latitude determinations were obtained. From the beginning of 1965 the results are sent to the international services at Mizusawa and Paris.

\section{BIBLIOGR A PHY}

Kostina, L. 1966, On the increase of precision of latitude calculations. Analysis of latitude observations, Trudy Tashkent astr. Obs., 12, 85-104.

\section{Résolutions proposées par la commission no. 19}

\section{Résolution}

(1) Les coordonnées du pôle instantané doivent être rapportées à une origine définie par les coordonnées initiales suivantes:

$\begin{array}{lr}\text { Mizusawa } & +39^{\circ} 8^{\prime} \\ \text { Kitab } & \text { ".602 } \\ \text { Carloforte } & 1.850 \\ \text { Gaithersburg } & 8 . .941 \\ \text { Ukiah } & 13.202 \\ & 12.096\end{array}$

Cette origine est appelée Origine Conventionnelle Internationale (OCI).

\section{Recommandations}

(1) Considérant que le problème de la dérive continentale est d'une grande importance et que les vitesses et les directions des dérives relatives des continents pourraient être confirmées et mesurées par des observations astronomiques, l'UAI recommande aux autorités concernées:

(a) que les stations existantes qui emploient soit des lunettes photographiques zénithales, soit des astrolabes Danjon et qui sont situées sur des parallèles suffisamment proches fassent des observations conjointes du temps et de la latitude. Cela implique que les mêmes étoiles appartiennent aux programmes d'observation de chacune des stations de la chaîne,

(b) que toutes les stations internationales des latitudes en fonction sur le parallèle nord de $39^{\circ} 8^{\prime}$ soient équipées de lunettes photographiques zénithales et qu'elles fassent avec ces instruments des observations régulières et conjointes du temps et de la latitude sans cesser les observations de la latitude avec les instruments visuels,

(c) qu'une chaîne similaire de lunettes photographiques zénithales soit établie dans l'hémisphère austral à une latitude voisine de $-34^{\circ}$, en Australie, en Afrique et en Amérique du Sud (en Argentine et au Chili), 
(d) que les observatoires du Mont Stromlo, du Cap, de La Plata et de Santiago forment une chaîne d'astrolabes et fassent des observations conjointes du temps et de la latitude,

(e) que, lorsque de nouvelles stations sont établies, elles soient placées en des lieux tels qu'elles puissent faire partie d'une chaîne existante ou en former une nouvelle,

( $f$ ) qu'une distribution d'astrolabes régulière en latitude soit conservée afin d'étudier les dérives entre stations de latitudes différentes,

( $g$ ) que soit étudiée la possibilité d'utiliser des satellites artificiels et des cataphotes sur la lune, afin de mesurer la dérive des continents avec grande précision.

\section{Remerciements}

(1) La commission 19 de l'UAI, appréciant la qualité des travaux du Service International du Mouvement Polaire et la rapidité de la publication de leurs résultats, prie le Dr. S. Yumi, directeur de ce service, d'accepter l'expression de sa reconnaissance.

(2) La commission 19 de l'UAI, ayant pris connaissance des valeurs définitives des coordonnées du pôle pour la période critique 1941-1949 prie le Professeur T. Nicolini qui s'est chargé du travail difficile de leur calcul, d'accepter l'expression de ses remerciements. 